\title{
Educación para todos: La Teoría de las Inteligencias Múltiples de Gardner ${ }^{1}$
}

\author{
Gisela Ernst-Slavit ${ }^{2}$ \\ Washington State University
}

\begin{abstract}
En los últimos años la Teoría de las Inteligencias Múltiples formulada por Howard Gardner ha tenido un impacto tremendo en los salones de clase de escuelas primarias y secundarias en los Estados Unidos de Norteamérica. Gardner (1983) define inteligencia como una habilidad para resolver un problema o manufacturar un producto valorado por lo menos en un contexto cultural. Esta definición extiende nuestra comprensión de inteligencia más allá de las reconocidas inteligencias linguiística y lógico-matemática, para incluir otras inteligencias como es el caso de la espacial, musical, física-cinestética, naturalista, interpersonal, e intrapersonal. Esta nueva manera de conceptualizar la inteligencia humana tiene profundas implicaciones para los educadores cuya labor deberá involucrar la identificación y nutrición de los diferentes talentos que traen consigo todos los estudiantes.

Palabras claves: inteligencia, Howard Gardner, educación, pedagogía.
\end{abstract}

\section{Education for All: Gardner's Multiple Intelligences Theory}

In the last years the Theory of the Multiple Intelligences developed by Howard Gardner has had a tremendous impact in elementary and secondary classrooms in the United States. Gardner (1983) defines intelligence as the ability to solve a problem or fashion a product that is valued in one or more cultural settings. His definition expands our understanding of "intelligence" beyond the familiar linguistic and logical-mathematical intelligences, to include the spatial, musical, bodily-kinesthetic, naturalist, interpersonal, and intrapersonal intelligences. This new way of conceptualizing human intelligence has profound implications for educators whose task needs to include the identification and nourishment of the different talents brought by al students.

Key words: Intelligence, Howard Gardner, education, pedagogy.

1 Este artículo se basa en la ponencia expuesta en el XI Education Seminar, Nishinomiya, Japón, noviembre, 1999.

2 Doctora de la Universidad de Florida, profesora asociada en Washington State University. Sus áreas de investigación incluyen el estudio etnográfico y sociolinguístico de contextos educacionales bilinguies o multilingües. El trabajo de la Dra. Ernst-Slavit ha sido publicado en numerosos libros y revistas. Correo electrónico: gernst@wsu.edu. 

"Educadores deben diversificar sus metodologías educativas para así poder alcanzar a la diversidad de personalidades que anidan en el salón de clase", dijo el profesor de Harvard, Howard Gardner a los participantes durante una convención en Milwaukee en 1997 (WEAC, 2001). Aduciendo que inteligencia es la habilidad para resolver problemas o la habilidad para hacer cosas valoradas por lo menos en una cultura, Gardner (1983) propuso una nueva definición de inteligencia que está creando una revolución en las aulas y escuelas en los Estados Unidos. En su libro Frames of Mind, publicado originalmente en 1983, Gardner primero concibió la Teoría de las Inteligencias Múltiples (T.I.M.) como una manera de extender y profundizar nuestra comprensión de "inteligencia" más allá de las reconocidas inteligencias linguística y lógico-matemática (que tradicionalmente enfatizan la lectura, la escritura y las matemáticas), para incluir otras.inteligencias como es el caso de la espacial, musical, física-cinestética, naturalista, interpersonal, e intrapersonal. Esta nueva manera de conceptualizar la inteligencia humana tiene profundas implicaciones para educadores cuya labor, por ende, deberá involucrar la identificación y nutrición de los diferentes talentos que traen consigo los estudiantes. La Teoría de las Inteligencias Múltiples confirma las sospechas de muchos educadores, de que todos los niños tienen algún tipo de talento.

\section{Perspectiva tradicional del cociente intelectual: los tests de inteligencia}

Antes de discutir la Teoría de las Inteligencias Múltiples de Gardner, sería interesante revisar brevemente las perspectivas tradicionales de la inteligencia (revisar, por ejemplo, Benjamin, 1997; Sternberg y Lubart, 1995; Sternberg, 1996; Yam, 1998). En 1905, Alfred Binet y Teodoro Simon desarrollaron la escala de Binet-Simon en París. El 
gobierno Francés comisionó a Binet y Simon para la creación de un test, para identificar a aquellos estudiantes con más oportunidades para sobresalir en el colegio y cuyos problemas de aprendizaje demandaran educación terapéutica. En 1930, Lewis Terman revisó esta prueba de inteligencia que luego se llamó el Test de Inteligencia de Stanford-Binet. Aquí, por primera vez, se introdujo el concepto de un cociente intelectual (C.I.). El C.I. es el resultado de la correlación entre la edad mental determinada por el test y la edad cronológica que generalmente es expresada como un cociente multiplicado por 100.

Actualmente, los tests de inteligencia de Stanford-Binet y el WISC (Wechsler Intelligence Scale for Children) son los tests más comúnmente utilizados para medir y clasificar inteligencia. Estas pruebas miden principalmente la habilidad de un individuo en las áreas lingüística y lógico-matemática y, en menor grado, la habilidad de completar algunas tareas visuales y espaciales. Es por ello que no es nada inusual encontrarse con personas con talentos notables, como, por ejemplo, el maestro de ajedrez, el conductor de orquesta, el atleta, que no son identificados por los métodos tradicionales de evaluar inteligencia. Peor aun, muchas de estas personas con talentos extraordinarios no solo no llegan a sobresalir en el colegio sino, por el contrario, fracasan. Consideremos los siguientes casos: Albert Einstein comenzó a hablar a los cuatro años y a leer a los siete. El maestro de música de Beethoven sugirió que él no tenía ningún talento para ser compositor. Leon Tolstoi no pudo completar su educación universitaria. Walt Disney fue despedido por el editor de un periódico porque "no tenía buenas ideas". Los maestros de Tomás Edison le dijeron que no tenía talento para aprender. Al famoso científico alemán, Werner von Braun, lo descalificaron en álgebra en la secundaria. Isaac Newton tuvo muy malas notas en la escuela primaria. ¿Cómo es posible que estos destacados personajes hayan tenido tantos problemas con los estudios? ¿Cómo es posible que personas con C.I. de 160 terminan trabajando para personas con C.I. de 100 ? 
La Teoría de las Inteligencias Múltiples de Gardner

Estas interrogantes pueden ser respondidas parcialmente si analizamos el concepto de inteligencia humana desde la perspectiva de Gardner.

\section{Las ocho inteligencias de Gardner: una nueva Teoría sobre la Inteligencia}

La Teoría de las Inteligencias Múltiples de Gardner claramente indica que el C.I. no se debe medir en términos inequívocos como cuando consideramos peso, altura, o presión de sangre. Es un error de tremendas proporciones, sostiene Gardner, asumir que el C.I. es una sola dimensión estática que puede ser medida con pruebas de lápiz y papel (Gardner, 1983; Gardner y Hatch, 1989). Gardner sostiene que no se trata de saber cuán inteligente una persona es sino cómo esa persona manifiesta su inteligencia. Como seres humanos todos nosotros tenemos un repertorio de habilidades para resolver diferentes géneros de problemas. Gardner (1983) define inteligencia como una habilidad para resolver un problema o producir un producto valorado en por lo menos un contexto cultural. En otras palabras, la inteligencia varía de acuerdo con el contexto. Un ejemplo de la importancia del contexto es el siguiente: si uno se quedara solo en la selva amazónica con un nativo de la tribu de los Yanomamos sin comida o agua, sería el nativo Yanomano el inteligente pues él sabría sobrevivir en ese ambiente. Si ese mismo nativo es trasladado a una escuela en Lima o Vancouver y le pedimos que asista a un grupo de niños estudiando divisiones y multiplicaciones, entonces la situación es muy distinta. Problemas, en este sentido, pueden ser planteados en diversas áreas como, por ejemplo, terminar un cuento, guiarse por las estrellas, anticipar una jugada de ajedrez, o arreglar los frenos de la bicicleta. Asimismo, los productos pueden involucrar el desarrollo de teorías científicas, composiciones musicales o campañas políticas exitosas.

Para desarrollar esta teoría, Gardner (1983) consideró perspectivas derivadas de neurobiología complementadas por otros campos como son la psicología, antropología, filosofía e historia. Asimismo, 
Gardner (1983) repasó la evidencia extensiva de una gama amplia de fuentes dispares. Analizó estudios de niños prodigios, individuos dotados, pacientes con daños cerebrales, niños normales, adultos normales, expertos en diferentes ramos de trabajo e individuos de culturas diversas. El resultado fue su influyente libro Frames of Mind (Gardner, 1983) donde manifestó que la inteligencia no es una habilidad innata que controla desde la creatividad hasta las habilidades para resolver problemas. Su teoría no cuestiona la existencia de una inteligencia general, sino sugiere que existen inteligencias no consideradas en este cociente llamado C.I. Más aun, sostiene que la inteligencia en el ser humano está localizada en diferentes áreas del cerebro que están íntimamente interconectadas, que son interdependientes - aunque cuando es necesario pueden trabajar independientemente de las otras - y que pueden ser estimuladas y desarrolladas en condiciones adecuadas (Gardner, 1983, 1993a, 1993b, 1994, 1999; Gardner, Kornhaber y Wake, 1996). Como es de esperarse, las conclusiones de Gardner sacudieron a la comunidad educativa quienes durante muchos años trabajaron, sin cuestionamiento alguno, con la noción de inteligencia como una única variable que puede ser medida con certeza con pruebas que solo utilizan lápiz y papel como son el StanfordBinet y el WISC.

En la publicación de 1983, Gardner perfiló siete inteligencias distintas: lingüística, lógico-matemática, espacial, musical, física-cinestética, interpersonal e intrapersonal. Luego, en 1995, sus investigaciones le sugirieron la existencia de una octava inteligencia, la naturalística (Gardner, 1995). ${ }^{3}$

3 Actualmente Gardner está considerando la posibilidad de agregar una novena inteligencia llamada existencial. Esta inteligencia se refiere a la habilidad de reflexionar sobre cuestiones existenciales: quiénes somos, por qué estamos en este mundo, cómo llegamos aquí. Esta inteligencia es característica de aquellos que analizan profundamente la realidad inmaterial y cuestionan asuntos donde no hay respuestas concretas. Es muy posible que este tipo de inteligencia se encuentre en teólogos, filósofos y gente espiritual. 
A continuación será definida cada inteligencia y serán presentados ejemplos de personajes reconocidos en el ámbito mundial.

Inteligencia lingüística: la habilidad de leer, escribir y comunicar con palabras. Escritores, periodistas, poetas y cómicos son ejemplos obvios de personas con inteligencia lingüística. Ejemplos famosos: Gabriela Mistral, Octavio Paz, Abraham Lincoln.

Inteligencia lógico-matemática: la habilidad razonar y calcular, pensar y organizar objetos en una manera lógica, sistemática. Estas son las habilidades muy desarrolladas en ingenieros, científicos, contadores, detectives y miembros de la profesión legal. Ejemplos famosos: Luis Alberto Sánchez, John Dewey, Maria Reiche.

Inteligencia espacial: la habilidad de poder comprender y expresar las imágenes visuales y espaciales. Esta habilidad se encuentra en arquitectos, artistas, escultores, marineros, fotógrafos y especialistas en planeamiento. Ejemplos famosos: Teodoro Núñez Ureta, Akira Kurosawa, Pablo Picasso.

Inteligencia musical: la habilidad de hacer o componer música, cantar bien o entender y apreciar la música, llevar el ritmo. Es un talento obviamente disfrutado por músicos, compositores e ingenieros de grabación. Pero la mayoría de nosotros tenemos un fundamento de inteligencia musical que se puede desarrollar. Pensemos cuán fácil se aprende cuando el mensaje va acompañado por un tintineo o una rima. Ejemplos famosos: Mozart, Plácido Domingo, Chabuca Granda.

Inteligencia física-cinestética: la habilidad de utilizar el cuerpo humano para resolver problemas, crear productos o transmitir mensajes y emociones. Una habilidad desplegada en el atletismo, la danza y el drama. En esta categoría se pueden incluir a cirujanos y a otras muchas personas quienes teniendo talentos con las manos no valoran esta forma de inteligencia. Ejemplos famosos: Marcel Marceau, Ana Pavlova, Michael Jordan. 
Inteligencia interpersonal: la habilidad de conectarse y trabajar eficientemente con otras personas, desplegar empatía y comprensión y comprender motivaciones y metas. Esta es una inteligencia vital humana desplegada por educadores ejemplares, jefes religiosos, terapeutas, políticos, y personas en el área de ventas. Ejemplos famosos: Mahatma Gandhi, Madre Teresa, Martin Luther King.

Inteligencia Intrapersonal: La habilidad de poder auto-analizarse, reflexionar, de ser contemplativo y evaluar calladamente las acciones y sentimientos más profundos, la capacidad de conocerse a sí mismo. Filósofos, consejeros y personas notables en muchos campos tienen esta forma de inteligencia. Ejemplos famosos: Sigmud Freud, Platón, Carl Rogers.

Inteligencia Naturalista: En 1995 Gardner decidió agregar una octava inteligencia, la naturalista. Esta inteligencia se refiere a la habilidad para reconocer flora y fauna, explorar y entender el mundo natural y usar estos conocimientos para mejorar cultivos o desarrollar las ciencias biológicas. Granjeros, botánicos, biólogos, activistas ecológicos. Ejemplos famosos: Charles Darwin, John James Audubon.

\section{Inteligencias múltiples en el salón de clase}

Muchos educadores en los Estados Unidos utilizan cotidianamente la Teoría de las Inteligencias Múltiples en el salón de clase. Un número creciente de maestros en las escuelas primarias y secundarias han desarrollado metodologías educativas y curricula basados en los principios de Gardner (e.g., Ernst-Slavit, Wenger y Statzner, 1998; Ernst-Slavit, Wenger y Krumweide, 2000). Si, además, consideramos la proliferación de libros y materiales acerca de las aplicaciones en el aula de la Teoría de Gardner disponible en librerías (e.g., Armstrong, 1994; Burke, 1993; Campbell, Campbell y Dickinson, 1999; Haggerty, 1995; Lazear, 1991, 1994, 1998; Nicholson-Nelson, 1998), conferencias profesionales, y en el Internet, podemos decir que en los Estados 
Unidos, la teoría de las inteligencias múltiples ha tenido un impacto enorme en los salones de clase de escuelas primarias y secundarias.

Pero implementar la Teoría de las Inteligencias Múltiples en el salón de clase no es tan fácil sobre todo en escuelas que utilizan programas de aprendizaje y estándares nacionales rígidos y donde los maestros están bajo presión por elevar el rendimiento de sus alumnos en pruebas estandarizadas en el ámbito regional o estatal. La implementación de la Teoría de las Inteligencias Múltiples requiere una reestructuración de las formas de enseñanza así como de la manera de asesorar el aprendizaje. La magnitud de los cambios generados por el uso de esta teoría dependerá de la habilidad del docente para reestructurar sus actividades y de la flexibilidad del sistema educativo para aceptar los cambios. Los obstáculos incluyen la falta de tiempo y esfuerzo. Se necesita tiempo para poder aprender nuevas maneras de pensar y organizar la enseñanza. Además, no es tan fácil cambiar la manera de pensar de muchos maestros que tradicionalmente preguntan ¿Cuántas páginas hemos repasado?, a preguntar: ¿Qué tan bien han entendido los estudiantes los conceptos y desarrollado su potencial?

A pesar de los obstáculos y dificultades que un cambio de esta magnitud representa para los maestros, muchos educadores en los Estados Unidos han volcado sus esfuerzos para poder planificar e implementar la currícula sobre la base de la propuesta de Gardner. Wilson (1998) sugiere, basada en sus entrevistas con muchos docentes, que la clave más importante para entender la masiva aceptación de esta nueva teoría, es la necesidad que tienen los maestros de crear un contexto educativo más apropiado que estimule y fortalezca las capacidades individuales de cada estudiante. Esta necesidad de cambio, aducida por muchos docentes, se manifiesta principalmente en cuatro áreas diferentes: 
- La TIM promueve comprensión y apreciación de las capacidades individuales entre los estudiantes.

- La TIM crea salones de clase donde se refuerza la autoestima en cada estudiante y donde los estudiantes desarrollan un poderoso sentimiento de pertenencia.

- La TIM desarrolla la capacidad intelectual y la motivación, y

- La TIM estimula a los educadores para que utilicen una variedad de estrategias educativas.

En otras palabras, la Teoría de las Inteligencias Múltiples ha sido recibida con los brazos abiertos en las aulas de los Estados Unidos porque ayuda a los educadores a reconocer y a aceptar las fortalezas y potenciales de cada estudiante; al mismo tiempo, refleja las creencias y filosofías profundamente arraigadas en padres de familia y maestros que todos los niños están dotados de habilidades y talentos, por ende, la misión del centro educativo es el desarrollo integral de cada niño.

A medida que los maestros mejoren en la comprensión e implementación de la teoría, de esa misma manera van a comprender y aceptar las diferencias individuales, valorar los talentos de todos y cada uno de los alumnos, y conocer y respetar la diversidad.

\section{Implicaciones}

Varias implicaciones con respecto al aprendizaje y a las técnicas educativas se deben tomar en consideración. Primero, la teoría declara que las ocho inteligencias se requieren para funcionar productivamente en la sociedad. Es por ello que educadores deben pensar en cada inteligencia como igualmente importante. Esta perspectiva está en gran contraste con los sistemas de educación tradicionales que típicamente enfatizan el desarrollo y la utilización de las inteligencias lingüística y lógico-matemática. Esto significa que los educadores 
deberán reconocer la diversidad intelectual y ofrecer experiencias de aprendizaje donde cada inteligencia sea valorada, estimulada y desarrollada.

Otra implicación se refiere a la necesidad de los maestros de planificar e implementar la curricula valiéndose de una amplia gama de recursos, para llegar a esas ocho inteligencias. Por ejemplo, en una clase de Inglés en el tercer grado, al enseñar una unidad sobre la casa (The House) se pueden incluir una variedad de actividades. Primero, los estudiantes dibujan una casa imaginaria en un país de habla inglesa (visual/espacial); los estudiantes imaginan quienes serían los ocupantes de la casa y cuales serían sus actividades cotidianas (intrapersonal); encuestan a los compañeros de clase acerca de los diferentes tipos de muebles en sus respectivos hogares y representan con una gráfica los resultados (lógico-matemática); preparan y prueban una ensalada de verduras típica norteamericana con tomates, lechuga, cebollas, zanahorias y apio (naturalista); dan comandos, tal como Abre la puerta y Cierra la ventana y ejecutan las acciones correspondientes (física-cinestética); cantan una canción acerca de la casa (musical); aprenden nuevas palabras sobre la casa y su contenido (lingüística); y en pares practican preguntas como ¿Dónde está el tejado? y ¿Quién está en la cama? (interpersonal). Este género de presentación no solo despierta el entusiasmo entre los estudiantes sobre el aprendizaje, pero permite que el maestro refuerce el mismo material en una variedad de estilos y con una variedad de materiales. Como resultado de la activación de una amplia gama de inteligencias, la enseñanza puede facilitar una comprensión más profunda de la materia.

Al nacer, todo individuo posee estas ocho inteligencias. No obstante, cada estudiante vendrá al aula con unas inteligencias más desarrolladas que las otras. Esto significa que cada niño tendrá un patrón único de fortalezas y debilidades. Estos patrones determinan cuán fácil (o difícil) le va resultar al estudiante aprender cuando la información se presenta de una manera particular. Este tipo de particularidad en el aprendizaje se refiere comúnmente como estilos de aprendizaje. 
En un salón de clase se pueden hallar muchos estilos de aprendizaje; es por ello que es imposible e impráctico que un maestro pueda acomodar en cada lección toda una gama de estilos de aprendizaje. No obstante estos obstáculos, el maestro puede ayudar a sus estudiantes a que reconozcan y utilicen sus inteligencias más desarrolladas para asistir en la comprensión de un asunto que normalmente emplea sus inteligencias menos desarrolladas (Lazear, 1998). Por ejemplo, el maestro puede sugerir que un estudiante dotado de una muy desarrollada inteligencia musical, aprenda sobre el arte del Renacimiento vía la composición de una canción acerca de la vida de Leonardo, Botticelli, Miguel Angel, Rafael, y otros o sobre sus respectivos trabajos desplegados en la Galeria Uffizi en Florencia.

\section{Conclusiones}

Docentes y escuelas tienen como rol fundamental el de ayudar a todos los niños a ser buenos estudiantes y miembros productivos de la sociedad. La Teoría de las Inteligencias Múltiples ofrece una sólida fundación teórica para reconocer los diferentes talentos y habilidades de los estudiantes. En lugar de ignorar o negar estas diferencias bajo la creencia de que todos los estudiantes tienen o deben tener, las mismas dotes y habilidades mentales, el sistema educativo deberá proveer a cada estudiante con oportunidades para el aprendizaje que maximicen sus potencialidades intelectuales. Inteligencia es simplemente un sistema de habilidades y potencialidades. Como educadores, podemos ayudar a todos nuestros estudiantes a desarrollar y mejorar su inteligencia si les enseñamos a conocer y utilizar tanto sus fortalezas como la manera de superar sus debilidades. 


\section{Referencias}

Armstrong, T. (1994). Multiple Intelligences in the Classroom. Alexandria, VA: ASCD.

Benjamin, L.T. (1997). A History of Psychology. New York: McGrawHill.

Burke, K.A. (1993). The Mindful School: How to Assess Authentic Learning. Palatine: Skylight Training and Publishing.

Campbell, L., Campbell, B. y Dickinson, D. (1999). Teaching and Learning Through Multiple Intelligences. Boston, MA: Allyn and Bacon.

Ernst-Slavit, G., Wenger, K., y Statzner, E. (1998). Compañeros: A teacher preparation partnership model for teaching FLES. Hispania, 81, pp. 379-391.

Ernst-Slavit, G., Wenger, K., y Krumweide, L. (2000). Preparing children for a global society: Integrating foreign language in the elementary school. In F.W. Parkay (Ed.). Curriculum Planning: A Contemporary Approach (7. ${ }^{a}$ edición) (pp. 356-363). Boston, MA: Allyn and Bacon.

Gardner, H. (1983). Frames of Mind: The Theory of Multiple Intelligences. Nueva York: Basic Books.

Gardner, H. (1993a). Multiple Intelligences: The Theory in Practice. Nueva York: Basic Books.

Gardner, H. (1993b). The Unschooled Mind: How Children Think \& How Schools Should Teach. Nueva York: Basic Books.

Gardner, H. (1994). Creating Minds: An Anatomy of Creativity Seen Through the Lives of Freud, Einstein, Picasso, Stravinsky, Eliot, Graham, and Gandhi. Nueva York: Basic Books.

Gardner, H. (1995). Are There Additional Intelligences? The Case for the Naturalist Intelligence. Harvard Project Zero. Cambridge, MA: President and Fellows of Harvard College.

Gardner, H. (1999). The Disciplined Mind: What All Students Should Understand. Nueva York: Simon y Schuster.

Gardner, H., y Hatch, T. (1989). Multiple Intelligences go to school. Educational Researcher, 18 (8), pp. 4-10. 
Gardner, H., Kornhaber, M., y Wake, W. (1996). Intelligence: Multiple Perspectives. Fort Worth, TX: Harcourt Brace.

Haggerty, B. (1995). Nurturing Intelligences: A Guide to Multiple Intelligences Theory and Teaching. Toronto: Addison-Wesley.

Lazear, D. (1991). Eight Ways of Knowing: Teaching for Multiple Intelligences. Pallatine: Skylight Training and Publishing.

Lazear, D. (1994). The Rubrics Way: Using MI to Assess Students' Understandings. Tucson: Zephyr Press.

Lazear, D. (1998). Multiple Intelligence Approaches to Assessment: Solving the Assessment Conundrum/Grades K-12. Tucson: Zephyr Press.

Nicholson-Nelson, K. (1998). Developing Students' Multiple Intelligences. New York: Scholastic.

Sternberg, R. J., \& Lubart, T. I. (1995). Defying the crowd. Nueva York: Free Press.

Sternberg, R. J. (1996). Successful intelligence. New York: Simon y Schuster.

WEAC (Wisconsin Education Association Council). Teachers Should Diversify Approaches to Teaching, Gardner Says. Recuperado el 13 de setiembre de 2001 http://www.weac.org/aboutwea/ conven $97 /$ gardner $2 . \mathrm{htm}$.

Wilson, L.O. (1998). What's the big attraction? Why teachers are drawn to using

Multiple Intelligence Theory in their classrooms. New Horizons for Learning Electronic Journal, 3(5). Retrieved from: http:// www.newhorizons.org/trm.lwilson 1.html.

Yam, P. (1998). Intelligence considered. Scientific American, 9(4), 611 . 